\title{
Oral Squamous Cell Carcinoma in the Young Patient
}

\author{
Priya Sivarajah (Meds 2016) \\ Faculty Reviewer: Dr Kevin Fung, MD, FRCSC (Department of Otolaryngology)
}

\section{INTRODUCTION}

Oral cancer is primarily a disease seen in elderly men (50-80 years of age) with a decades-long history of smoking, and consumption of over 20 cigarettes and $100 \mathrm{~g}$ of alcohol a day. ${ }^{1}$ Its presentation in younger patients ( $<35$ years) is fortunately rare, with an incidence between $0.4 \%$ and $5.5 \% .{ }^{1}$ However, when cases do present, they are often misdiagnosed, leading to inappropriate delays in treatment, and worsening prognoses. ${ }^{2}$ This bears significant implications for young patients, with the potential for long-term functionally debilitating sequelae. With this in mind, we report a case of oral tongue squamous cell cancer (OTSCC) in a young Caucasian female, with a focus on her diagnoses and subsequent post-surgical quality of life.

\section{CASE REPORT}

A 25-year old Caucasian female presented to a dentistry clinic, complaining of a left-sided lesion on her tongue of 13 months duration, which had markedly increased in size within the past month. She reported an impact on her speech and eating patterns, and expressed worry about a malignant etiology. The patient had initially believed the lesion to be a chancre sore, but decided to seek help as the lesion had continued to grow.

The patient had an intermittent smoking history of an unspecified duration, during which she smoked up to 5-10 cigarettes daily. She also had a five-year history of drinking, a period through which she consumed 8 drinks per day, for four days a week. She denied experiencing night sweats or fevers, although she reported losing weight from a markedly reduced food intake. Her paternal grandfather had died from metastatic esophageal cancer, and her maternal grandfather was diagnosed with colon cancer.

The dental practitioner reassured her (allaying her fears of cancer), filed down her teeth, and scheduled a follow-up appointment in 2 weeks' time, during which her presentation changed significantly. She started experiencing constant headaches, and the lesion grew purulent, significantly affecting her ability to eat. Finding that conservative management with salt and water, as well as peroxide was futile, she visited her university walk-in clinic to receive antibiotics, noting once again that the physician failed to discuss the possibility of a malignant etiology. Re-visiting her dental practitioner at her follow-up appointment, the rapid change in presentation quickly prompted a referral to a dental specialist at an academic care centre. Although the appointment wasn't for another four months, the exponential growth of the tumor led the patient to successfully reschedule the appointment to a closer date, within two months' time. A physical examination prompted the specialist to quickly refer the patient to an otolaryngologist, who saw her within the week.
The surgeon prescribed her Tylenol-Codeine \#3, which she took with Ibuprofen and Acetaminophen for pain management. Her diet was reduced to soft meals, consisting of pudding, apple sauce and yogurt. Three weeks later, after confirmation through biopsy of a T2NO squamous cell carcinoma of the oral tongue, the patient underwent a selective neck dissection. A left-sided hemiglossectomy removed the carcinoma, and a fasciocutaneous radial forearm free flap from the right arm replaced the defect. The skin defect was replaced using a graft from the right hip. A tracheostomy was performed, and a feeding tube was inserted. The absence of metastasis precluded the need for chemotherapy or radiation treatments. The patient remained hospitalized for an additional two weeks.

Post-surgical QoL issues circled around dysfunction of the left shoulder muscles, sensitivity of the left sided face and right arm, and with problems inherent in having a tracheostomy and feeding tube. The patient's face was numb for an unspecified duration post-surgery, and her tongue was significantly swollen for a week. In addition to the cast on her right arm, and IV's in her left, communication (both verbal and written) during that week was significantly impaired.

The patient received integrated care from a team of inter-professional allied healthcare workers. A respiratory therapist aided her in coughing exercises to prevent aspiration pneumonia from her tracheostomy; a physiotherapist aided her transition off the tracheostomy, familiarizing her with breathing exercises, and encouraging the change from bed-rest to ambulation; a speech language pathologist performed pre- and post-op assessments to evaluate standard articulation and swallowing; a nutritionist made recommendations post-surgery to assist the patient's food intake; and a home care nurse assisted the patient in showering and re-dressing the cast on her right arm. The patient had pureed meals for 2 months, and continued her pain medications for one month post-surgery. With the assistance of her parents and her healthcare team, the patient was able to recover and return to school for the next academic year. Following surgery, the patient has had annual follow-up appointments with no evidence of recurrent disease to date. She subsequently quit smoking, and has since given birth to her first child.

The patient still suffers from ongoing QoL issues. The patient has a persistent dry mouth, and has reduced sensation of her left-sided tongue, occasionally not noticing when she bites on it. She is unable to differentiate between soft and hard bites, and has changed her eating patterns, shunting food from the right side of her mouth to make use of her remaining taste buds. This has spawned dentition problems, including right-sided gum loss and left-sided gum growth. She has recurrent neck and jaw pain, which she treats with Tylenol. She has also noticed changes in her facial features, with her left-sided face appearing 'droopier'. 


\section{DISCUSSION}

\section{EPIDEMIOLOGY}

Oral tongue squamous cell carcinoma (OTSCC) is the most common tumor in the oral cavity. ${ }^{2}$ Although the overall incidence of OTSCC has remained stable from 1975 to 2007, its incidence has been increasing in women, specifically in young, Caucasian females aged 18-44 years. ${ }^{1}$ Unfortunately, as is evident in the case presentation, there is a stigmatized approach to younger female patients with suspicious oral lesions. Raising awareness on these rising incidence levels will alert healthcare practitioners to atypical presentations, and reduce any delays these patients may experience in receiving appropriate care.

\section{RISK FACTORS FOR YOUNGER PATIENTS}

Although the literature is uncertain and the topic still highly debated, it has been claimed that younger patients have more aggressive disease, with a higher incidence of local recurrence or regional lymph node involvement after treatment, and higher mortality rates. ${ }^{1}$ Young patients often present with little-to-no risk factors (alcohol, tobacco and betel nut exposure), or with a history of tobacco and alcohol intake significantly shorter in comparison to their older counterparts. Given this disparity, researchers have proposed other risk factors to account for the etiology of these oral cancers, such as immune deficiency states, genetic factors, dietary factors, and viral infections (such as herpes simplex virus and human papilloma virus). ${ }^{1} \quad$ The association between oropharyngeal cancer and HPV infection is controversial at best, and significant research has been focused on screening for specific virally-associated mutations of known tumor suppressors, oncogenes, and replication pathways. ${ }^{2}$ Unfortunately, no clear inciting pathway has been identified to this date.

\section{QUALITY OF LIFE}

Research into QoL issues for young oral cancer patients is a rather new and emerging concept. Thomas et al. performed a retrospective case series and questionnaire survey for patients treated for oral cancers during a 25-year period, when they were 40 years of age or less. They found that age at diagnosis and duration of follow-up did not correlate with overall QoL or health-related QoL. Approximately $77 \%$ of patients rated their overall QoL as outstanding, very good or good, with the key problematic domains lying within appearance, mood, salivation and shoulder function. Only radiotherapy adversely affected the overall QoL. ${ }^{3}$ In addition, a systematic review by Carranza et al., found that younger oral cancer patients show more emotional and role dysfunctions than older patients, and are at higher risk for psychological stress and an increase in symptoms-such as dry mouth-in the first post-treatment year. Marital status seems to be a protective prognostic factor for survival and recurrence in comparison to those who live alone, possibly due to better hygienic habits, and fewer delays in diagnosis and treatment. ${ }^{4}$

\section{CONCLUSIONS}

Although there is adequate research into QoL for oral cancer patients, most of the current literature solely investigates elderly patients with significant histories for known risk factors. There is a noticeable lack of research into QoL specific to younger oropharyngeal cancer survivors, namely OTSCC survivors. The rising incidence of these cancers in the younger population raises the need for further studies to be done, in order to ensure that younger patients return to a fruitful life with limited functional disabilities.

\section{REFERENCES}

1. Majchrzak E, Szybiak B, Wegner A, Pienkowski P, Pazdrowski J, Luczewski L, Sowka M, Golusinski P, Malicki J, Golusinski W. Oral cavity and oropharyngeal squamous cell carcinoma in young adults: a review of the literature. Radiol Oncol. 2014; 48(1): 1-10.

2. Goepfert RP, Kezirian EJ, Wang SJ. Oral Tongue Squamous Cell Carcinoma in Young Women: a Matched Comparison- Do Outcomes Justify Treatment Intensity? ISRN Otolaryngol. 2014: 529395.

3. Thomas L, Moore EJ, Olsen KD, \& Kasperbauer JL. Long-term quality of life in young adults treated for oral cavity squamous cell cancer. Ann Otol Rhinol Laryngol. 2012; 121(6): 395-401.

4. Eusebio Torres C, Cossío P, Guisado J, Aumente E, Pérez J. Assessment of quality of life in oral cancer. Med Oral Patol Oral Cir Bucal. 2008; 13(11): E735-41. 